\title{
L'improvisation, du fragment à la plénitude
}

\section{Tristan IKOR}

Résumé : Quel est le sens de ce geste, finalement assez étrange, qui consiste à vouloir (s')improviser en musique, jusqu'à vouloir se montrer improvisant ? C'est en résonnant de grand air que ses significations raisonnent, et qui s'abordent aussi en sabordant. Ici, le complexe d'identité dans son entier est bouleversé, comme mis à vif dans un amour du son délesté de musique : l'urgence s'éprouve au plus fin, sur la crête d'un temps prêt à tout moment à s'évaporer en une immédiateté aussi foncièrement nouvelle qu'ancestrale. Le spectacle d'improvisation apparaît dès lors comme une sorte de lieu de jonction entre la fête sacrée et l'intimité d'un art brut. A la rencontre de l'autre pourtant dénié, le rapport à soi de l'improvisateur en jeu retrouve sa mondanéité propre et son originalité souveraine.

Resumo: Qual é o sentido deste gesto, finalmente bastante estranho, que consiste em querer (se) improvisar em música, chegando a querer se mostrar improvisando? É com ares de superioridade que estes significados se apoiam, e se abordam mesmo quando se dissolvem 1 . Aqui, a identidade complexa em seu todo se perturba, como que trazendo à vida um amor ao som desprovido de musica: a urgência é provada mais minuciosamente, sobre a crista de um tempo prestes à se evaporar a qualquer momento num imediatismo fundamental e simultaneamente novo e ancestral. O espetáculo da improvisação aparece a partir daí como uma espécie de ponto de junção entre a festa sagrada e a intimidade de uma arte em estado bruto. Ao encontro do outra, portanto renegado, a relação do improvisador consigo mesmo quando em ação, encontra sua mundaneidade propria e sua originalidade soberana.

\footnotetext{
1 Atente-se para o jogo de palavras no original : s'abordent aussi en sabordant. [N.do Ed.]
} 
«L'unique lancer est un chaos, dont chaque coup est un fragment. $»^{2}$

Mes deux invités sont en face de moi. L'un est un improvisateur, l'autre est chercheur en sciences sociales. La transcription de cette discussion, prise en cours de route, pourrait commencer ici:

Comment définiriez-vous l'improvisation?

L'improvisateur: Simplement comme ce qui n'a pas été prévu à l'avance...

Le chercheur: ... mais si cette définition courante est juste, elle reste équivoque. La musique n'existe qu'apparue, elle est irréductiblement orale; comment dans ce cas prévoir totalement ce qu'elle va être? Même l'interprétation d'une oeuvre écrite possède une part d'improvisation. Puisque le temps ne se répéte jamais ${ }^{3}$, il est impossible de jouer deux fois la même phrase musicale de façon identique, même en respectant l'accentuation, les nuances, le tempo, etc... L'écriture ne fait que limiter ce qui sera improvisé: plus l'écriture sera précise, plus le jeu improvisé se fera dans les détails. II y a nécessairement une part d'improvisation en toute musique, l'écriture délimitant l'étendue de son terrain de jeu.

I: Il y a selon moi davantage complémentarité qu'opposition entre l'écrit et l'oral4: dans un processus de création, seule compte la musique apparue; qu'importe les outils utilisés. Ceci dit, la forme de cette complémentarité peut permettre de classer les musiques sans considération de style: une partition classique peut se rapprocher de

2 Gilles Deleuze, Logique du sens, Les éditions de minuit, Paris 1969, p. 75.

3 Daniel Charles, Le temps de la voix, Ed. J.-P. Delarge, Paris 1978, p. 58.

4 Denis Levaillant, L'improvisation musicale, Essai sur la puissance du jeu, Actes Sud, Arles 1996, p. 231. 
l'écrit mémorisé de certaines musiques de variétés, l'improvisation de jazz est aussi contraignante que celle de l'organiste (la grille de l'un rappelant les chiffrages de l'autre), l'oeuvre ouverte de la musique contemporaine peut s'apparenter à une improvisation qui s'autorise à piocher dans un répertoire, ou encadrée par une trame, ou encore à une composition utilisée comme tremplin à l'improvisation, toutes autant utilisées dans les musiques dites actuelles que dans le jazz moderne; la contrainte écrite peut aussi être ce que l'on accompagne en improvisant, que ce soit un texte, de l'image, une mise en scène ou une chorégraphie... Les musiques traditionnelles improvisent autour de leur socle culturel, plus ou moins contraignant, et ainsi le renouvelle. Jusqu'à l'écriture minimale qui consiste à ne prévoir que le fait de ne pas prévoir le contenu; dès lors, nous sommes dans une forme musicale particulière qu'on appelle musique improvisée, ou libre ou non-idiomatique5. Mais il s'agit alors d'une volonté consciente d'improviser, d'une posture, qui ne se traduit pas nécessairement par une improvisation au sens plein. Même dans cette forme, il y a toujours du prévisible, selon le répertoire intérieur du musicien, alors que l'improvisation pleine est création de savoir. La posture d'improvisateur correspond la plupart du temps à un travail de compositeur dans l'instant...

C: ... d'instant en instant, justement, et non dans l'instant. Seule l'improvisation pleine peut se dire dans l'instant. Dans le jeu improvisé comme dans la posture d'improvisateur, ne pas prévoir à l'avance signifie ne pas prévoir avant que la musique n'apparaisse. Mais une fois dans l'urgence de la musique qui se déroule, chaque acte repose sur l'anticipation, qui est une forme de prédiction dans le temps court. II y a distanciation dans le temps entre la perception, la décision et la réaction effective: à partir de la musique qui existe déjà, et avec le souvenir de ce qu'elle a été, on imagine ce qu'on désire entendre avant de le produire. L'anticipation caractérise la présence de l'interprète comme de

5 Derek Bailey, L'improvisation: Sa nature et sa pratique dans la musique, Outre-Mesure, Paris 1999, p. 4. 
l'improvisateur; et c'est dans la qualité de cette présence qu'une improvisation fait sens.

I: Être présent, c'est à la fois faire et se regarder faire, avoir conscience du geste désiré et réaliser ce geste; la concentration se focalise sur ce que je veux être en lien avec ce que je fais. L'idée de présence réunit actorialisation (être présent), temporalisation (au temps présent) et spatialisation (être là): je suis, ici, maintenant. Elle ne saurait supporter une expression inconsciente non maîtrisée, pas plus qu'une errance dans la pensée, désincarnée.

C: Mais l'urgence ne fait pas l'immédiateté. L'improvisation au sens plein transgresse cette présence, elle refuse d'anticiper.

Si je vous comprends bien, il y a le jeu improvisé nécessairement présent dans toute expression orale, la volonté d'improviser ou la posture d'improvisateur qui construit une forme musicale particulière, et l'improvisation pleine, immédiate et transgressive.

C: En effet, on peut délimiter l'improvisation selon la quantité d'imprévisible que la musique contient, jusqu'à la forme qui se veut totalement improvisée. Mais on peut aussi l'appréhender de façon plus stricte, selon la qualité d'immédiateté. Juger de cette qualité fait apparaître l'expérience de transgression qu'est l'improvisation pleine.

Mais pourquoi cette dernière est-elle une transgression?

I: Encore une fois, le mot est trompeur: il ne s'agit pas du tout d'un rapport aux règles musicales, qu'il s'agirait d'enfreindre pour improviser librement... Cette relation aux codes permet de concevoir l'appartenance d'une musique, elle ne parle que de mode quand la transgression est de l'ordre du droit ${ }^{6}$. Les codes de tel style ou de telle

$6 \quad$ Eric Landowski, Présences de l'autre: essai de socio-sémiotique II, PUF, Paris 1997, p. 123. 
époque peuvent devenir les interdits de tels autres; mais l'improvisation n'est pas un style et la transgression n'a que faire de la notion d'interdit.

C: La transgression est plutôt le mouvement qui inverse une propension. La transgression nous fait passer dans un autre mode d'existence, elle est un déplacement. L'improvisation pleine est une expérience qui nous transporte dans une autre forme de conscience de soi et du monde. Durant cette expérience radicale, aux limites du pensable, le temps n'est plus une succession de moments, d'états. Je suis au contraire dans l'immédiateté, qui est un passage permanent, insécable. Je vis mon devenir sans médiation. La posture d'improvisateur manipule la matière sonore dans l'urgence, c'est-à-dire en essayant de concevoir une succession de moments très précis, infimes; l'improvisateur qui n'improvise pas encore est en face du temps, en face du son, il se regarde jouer et se comprend comme un musicien face à un public, à un environnement... L'improvisation pleine fait entrer l'improvisateur dans le son, dans le devenir, elle démolit les distinctions entre énoncé et énonciation puisque je deviens littéralement le son, entre Moi et le monde puisque mon identité n'est plus limitée à mon enveloppe corporelle. Elle est une expérience de Vérité. II n'y a plus de choix conscients distanciés dans le temps de leurs réalisations, mais des décisions inconscientes et surtout nécessaires, qui se prennent en se faisant. Est improvisé ce qui devient par soi-même. Pour cela, il n'y a pas d'erreur dans l'improvisation pleine, seulement des échecs en posture dans le fait de parvenir à improviser. On peut aussi définir ce moment comme un oubli, ou un débordement.

I: Pourtant, je ne m'oublie pas à ce moment: je continue à entretenir une réflexivité sur moi-même, de même que j'utilise ma mémoire. II s'agirait au contraire d'une conscience aiguisée de soi dans l'instant. Je préfère l'idée de débordement. Par exemple, l'instrument comme prolongement de son corps est une image qui aide l'interprète; cette idée est réellement vécue dans l'improvisation pleine. 
C: Bien sûr, l'improvisation pleine ne s'apparente pas à l'inconscience, ou à une forme d'immédiateté caractérisée par l'absence de regard sur soi. L'improvisateur n'est pas l'esclave d'une quelconque force métaphysique, il n'est pas passif mais décide en tant que son, avec une perception et une conscience élargies et affinées. II a toujours besoin de ce regard élevé de la conscience. L'improvisation pleine n'annihile pas le pour-soi dans un pur en-soi, mais propose une expérience particulière qui réunit dans le même geste l'en-soi et le pour-soi, l'immédiat et le réflexif. II y a bien réflexivité, mais une réflexivité spécifique: étant donné que je suis le son, que l'être déborde dans son environnement, cette réflexivité se concentre sur soi en tant que monde. L'oubli n'est donc pas un oubli de soi, mais l'oubli des frontières habituelles du soi. En psychanalyse, l'oubli peut désigner ce moment fondateur où, par la prise de conscience de nos limites, nous oublions l'existence pré-langagière de conjonction avec le monde7. En ce sens, l'oubli de l'improvisation pleine est une sorte d'oubli d'oubli; ce qui n'équivaut pas à se souvenir, puisque l'oubli qui fonde notre identité est inoubliable, mais à découvrir une nouvelle forme d'existence. Cette dernière s'apparente à une forme d'existence première, mais enrichie de la mémoire de la connaissance de notre identité irréductible - il n'est pas ici question d'une mémoire considérée comme un stockage de données, mais d'une mémoire vive, organique. La pulsion invoquante et proprement musicale, définie par Alain Didier-Weill, serait justement cet appel à la transgression par la musique.

Pouvez-vous vraiment délimiter une telle expérience dans le réel?

I: Cette expérience existe indéniablement, ce sont les mots pour en parler qui manquent... Elle ne doit pas être confondue avec un simple moment d'émotion, ni même avec le moment où ça tourne, quand la

$7 \quad$ Alain Didier-Weill, Les trois temps de la Loi, Seuil, Paris 1995, p. 249. 
musique fonctionne - ici aussi, notre entendement a peu d'accroche, les mots manquent pour définir une sensation que tout musicien connaît pourtant, et la notation ne saurait transcrire avec justesse ce que sont, par exemple, le swing ou le groove. Ceci dit, cette sensation d'évidence, d'aisance dans le jeu ou de cohérence partagée (qui ne dépend pas de ce qui est joué mais de comment cela est joué), cela est selon moi un tremplin nécessaire à l'improvisation pleine: cette dernière ne saurait survenir alors que les musiciens n'ont pas trouvé un terrain d'entente qui fonctionne musicalement. Lorsque la musique les réunit enfin, un autre pas reste à faire pour connaître ce débordement.

C: L'improvisation pleine est justement une irruption de réel au sein d'un discours symbolique. L'adjectif plein résulte d'une analogie entre cette expérience musicale et la parole pleine définie par Lacan. Lorsque je suis seulement dans une posture cherchant à improviser, lorsque je compose dans l'urgence et à plus forte raison quand j'interprète une partition, je construis un discours symbolique, esthétique. L'improvisation pleine fait sortir du symbolique pour vivre une expérience réelle; l'éthique devient plus pertinente que l'esthétique pour comprendre ce moment qui, rappelons-le, est rare et particulièrement éphémère. Ce peut être deux secondes de vérité pour un concert entier... La posture d'improvisateur peut être considérée comme une esthétique de l'éthique, l'improvisation pleine comme l'expérience éthique de l'esthétique.

I: Je pense qu'il est préalablement nécessaire de bien saisir ce qui est en jeu dans la posture d'improvisateur, voire en toute forme d'improvisation, pour ensuite comprendre la spécificité de l'improvisation pleine.

Dans ce cas, revenons dans le pensable: qu'est-ce qui définit une posture d'improvisateur? 
I: J'aime cette notion de posture car elle évoque déjà les valeurs propres à l'improvisation, ainsi que la réalité de sa pratique. Pour Deleuze, la posture est un mode d'énonciation. A la base, la posture n'est que la position du corps dans l'espace - évidemment, ce positionnement est déjà significatif - la manière dont l'organisme affronte les stimulations du monde et se prépare à y réagir. Par cette notion, on conçoit donc déjà le jeu improvisé comme une relation entre soi et le monde, basée sur l'anticipation de la réaction à une perception. Mais par extension, nous parlons de posture pour désigner un état (une disposition morale de l'esprit ou une situation sociale), ou un comportement, une conduite. Or, la posture d'improvisateur est un concept qui réunit ces différentes acceptions, sans que l'une soit l'analogie de l'autre. Elle naît de la volonté d'improviser, volonté assumant la part d'inconscient qui la dépasse et attachée à penser ensemble corps et esprit, raison, sensibilité et inconscient, intéroceptif et extéroceptif, une conduite sociale et sa morale singulière, le temps et l'espace, la maîtrise de soi8 et le lâcher prise, le structurel et l'existentiel... Ainsi, l'improvisateur se positionne en tant que tel dans l'espace public, en voulant exprimer sa singularité physique et intellectuelle. Improviser révèle et constitue l'identité de l'improvisateur.

C: Cette façon de voir la création musicale est profondément politique, car elle représente un rapport à l'autre. L'improvisateur en acte construit un équilibre propre entre ses désirs et les attentes supposées de son environnement; entre son identité individuelle qui fonde sa

8 Michel Foucault, Histoire de la sexualité II, L'usage des plaisirs, Gallimard, Paris 1984 , p. 89 . Si la pratique de l'improvisation nécessite bien une maitrise de soi, celle-ci diffère de celle des philosophes grecs comme de la morale chrétienne car elle n'est pas un combat contre le désir. La posture est une maîtrise visant la surprise de soi, ayant pour but d'éviter ce qui gêne l'expression du désir. 
singularité et son identité collective qui fonde son appartenance ${ }^{9}$. Entre son Moi et son Soi 10 .

I: Cette distinction, couplée à celle opérant entre réflexivité et immédiateté, permet de concevoir des postures typiques, entre lesquelles tout improvisateur oscille. La présence que nécessite l'improvisation évite ces stéréotypes en assumant l'existence simultanée de désir et de besoin de reconnaissance, à la fois en-soi et pour-soi.

Mais que penser alors des postures qui refusent l'expression d'une identité au travers de la musique?

C: Paradoxalement, même celui qui tente de faire disparaître de sa musique tout processus d'identification représente une de ces postures identitaires, politiquement significative. C'est justement I'identité qui fait d'un son une musique. Pour cela, un amoureux du son comme John Cage peut être gêné par la musique'l. Mais sachant qu'écouter implique aussi des choix identitaires, un son ne devient-il pas musique dès lors que nous l'écoutons? En fait, dans cette posture particulière, l'expression de l'identité du créateur semble vouloir se faire discrète pour laisser plus de liberté à celle de celui qui écoute.

Mais nous ne sommes plus dans le jeu alors?

I: L'écoute fait partie du jeu improvisé, qui n'est ni le jeu d'acteur (l'improvisateur cherche au contraire à devenir ce qu'il pense être), ni le fait de produire un son (ne pas produire un son est déjà un choix musical). II n'est évidemment pas non plus le jeu qui, selon des règles préétablies, distingue les vainqueurs des vaincus. Le jeu improvisé est

9 Bernard Lamizet, Politique et identité, PUL, Lyon 2002, p. 6.

10 Eero Tarasti, Fondements de la sémiotique existentielle, L'Harmattan, Paris 2009, p. 110.

11 John Cage, Je n'ai jamais écouté aucun son sans l'aimer: le seul problème avec les sons, c'est la musique, La Main courante, La Souterraine 1994, p. 1. 
bien plus proche du jeu idéal que définit Deleuze12. A l'image du devenir, le jeu idéal est un chaos impensable dans lequel nous ne pouvons que le fragment. Le jeu improvisé est écoute et expression, et le complexe d'identité dont nous parlions peut aussi bien s'appliquer à l'un qu'à l'autre. Car l'écoute, si elle n'est pas créative, n'en est pas passive pour autant. Nous construisons notre environnement d'abord par élimination; l'écoute est une façon de sélectionner, et cette focalisation révèle et construit l'identité de celui qui écoute. Ainsi, nous développons chacun un pouvoir d'écoute différent, qui s'alimente et s'entretient. L'expression, elle, créera à partir de ce qui a été choisi comme monde par l'écoute. Le jeu improvisé, présent en toute forme d'oralité, englobe donc l'écoute qui élimine et l'expression créatrice. La qualité de l'écoute d'un improvisateur est aussi importante que sa capacité à s'exprimer. Le sens de la présence se comprend par la justesse du ressenti de l'instant, et l'improvisation est une interprétation du monde qui cherche à accorder au mieux ce qui est perçu avec ce qui est exprimé.

C: D'ailleurs et de façon imagée, l'écoute est aussi écoute de soi. En cela, la projection de ce que nous supposons être est déjà une interprétation politique du monde. De la même manière, l'expression est autant expression de soi qu'expression du monde tel que nous le comprenons.

Dans quelle mesure vous ne parlez plus uniquement de musique?

I: L'improvisation pleine, en fin de compte, ne saurait être limitée dans une pratique artistique particulière. II s'agit d'une expérience synesthésique, et rien ne devrait m'empêcher de danser ou de parler lorsque j'improvise. Encore une fois, ce n'est pas tant ce qui est exprimé qui importe ici que la façon et le fait même de s'exprimer.

12 Gilles Deleuze, op. cit., p. 74. 
C: Toutes ces idées m'intéressent finalement plus en elle-même que pour comprendre la musique, voire la création. Je me suis d'abord armé de réflexion pour comprendre l'improvisation musicale; aujourd'hui, cette dernière n'est qu'un tremplin, un exemple pratique qui me permet de mieux cerner ce qu'est l'improvisation en général et qui concerne beaucoup plus que la musique. Ce concept éclaire sans cesse ma recherche et affine ma méthodologie, il véhicule également tout un ensemble de valeurs. Par exemple, et de la même manière qu'une poésie ne saurait être bête sans en devenir mauvaise, la science peut s'enrichir d'une expression sensible de sa pensée, surtout lorsqu'elle cherche à comprendre les limites du rationnel, voire du pensable ${ }^{13}$. Tout langage possède une musicalité qui participe de sa signification: la compréhension n'est pas que raisonnée, le sens survient aussi par les sens. On pense aussi avec son corps. Autre exemple, l'improvisation appelle à reconsidérer le rapport de la pensée à l'écrit, ce qui explique le fait de préférer l'oral et la discussion à un écrit magistral et sans faille.

Mais vous devez savoir que nous n'existons pas plus que nous ne discutons actuellement: nous sommes les personnages fictifs d'un texte clos.

C: Vous dîtes cela car vous êtes également narrateur... Mais je ne suis pas censé connaître ce métalangage, car ceci est un jeu dans lequel mon rôle est de croire que nous parlons. Je pense en effet que l'auteur qui nous a créé ne considère pas son texte comme une discussion réelle; mais ce jeu de miroir entre le contenu de ce que nous disons et la forme de ce texte éclaire certaines idées, il est l'application méthodologique des premières conclusions d'une pensée sur l'improvisation: I'oral est un flux qui se disperse en tout sens, et nous ne pensons que par fragments. L'improvisation comme la pensée n'ont jamais le caractère fini d'une

13 Friedrich Nietzsche, Le gai savoir, Gallimard, Paris 1982, p. 113. 
écriture, elles refusent l'exhaustivité, comme la prétention à saisir totalement un objet, ou à épuiser une idée. Elles préfèrent l'idée de justesse à celle de perfection. Aussi, l'improvisateur comprend et utilise sa mémoire d'une façon particulière, qui peut être assimilée par la posture du chercheur. Mais au-delà de la recherche, l'idée d'improvisation et ce qu'elle véhicule arrosent de nouvelles significations et de nouvelles possibilités la vie en général. Elles peuvent aider à faire face à une maladie, à vivre différement avec soi-même. En fin de compte, l'idée d'improvisation concerne une philosophie de l'existence et de la connaissance - particulièrement de la connaissance de soi. Elle implique aussi un engagement politique, dans le sens où j'engage mon identité dans un rapport au collectif.

I: Je ne connais pas d'improvisateur qui considère sa pratique comme isolée de sa manière de vivre. Être improvisateur, ce n'est pas un métier mais un engagement total. On ne cesse pas d'improviser ou de s'entraîner à l'improvisation à la sortie du concert, on se construit en permanence; tout ce que je vis et jusqu'à ma façon de penser influe sur ma manière de jouer, de même que mes expériences musicales changent ma façon de concevoir le monde. Yoshi Oida travaille sa concentration en se lavant les dents ${ }^{14}$. Les moments de vie improvisée acceptent sans se résigner le déroulement du temps, sans regret ni espoir, sans désespoir ni satisfaction. Une posture d'improvisateur peut s'adopter pour marcher, se laver, faire l'amour, dans la manière de penser, dans une discussion entre amis ou dans une relation de couple...

C: Reste à savoir ce qu'est improviser en dehors de toute médiation artistique, si cela est possible. L'improvisation ne se suffit pas de l'écoute, et dès lors que nous doublons une expérience esthétique d'une expression personnelle, ne rentrons-nous pas dans la création artistique?

14 Yoshi Oida, L'acteur rusé, Actes Sud, Arles 2008, p. 95. 
Bien sûr, la discussion ne s'arrête pas là. Aussi, trois petits points sont toujours préférables à un point final...

\section{Références bibliographiques}

Bailey, Derek, L'improvisation: Sa nature et sa pratique dans la musique, Outre-Mesure, Paris 1999

Cage, John, Je n'ai jamais écouté aucun son sans l'aimer: le seul problème avec les sons, c'est la musique, La Main courante, La Souterraine 1994

Charles, Daniel, Le temps de la voix, Ed. J.-P. Delarge, Paris 1978

Deleuze, Gilles, Logique du sens, Les éditions de minuit, Paris 1969

Didier-Weill, Alain, Les trois temps de la Loi, Seuil, Paris 1995

Foucault, Michel, Histoire de la sexualité II, L'usage des plaisirs, Gallimard, Paris 1984

Lamizet, Bernard, Politique et identité, PUL, Lyon 2002

Landowski, Eric, Présences de l'autre: essai de socio-sémiotique II, PUF, Paris 1997

Levaillant, Denis, L'improvisation musicale, Essai sur la puissance du jeu, Actes Sud, Arles 1996

Nietzsche, Friedrich, Le gai savoir, Gallimard, Paris 1982

Oida, Yoshi, L'acteur rusé, Actes Sud, Arles 2008

Tarasti, Eero, Fondements de la sémiotique existentielle, L'Harmattan, Paris 2009 
